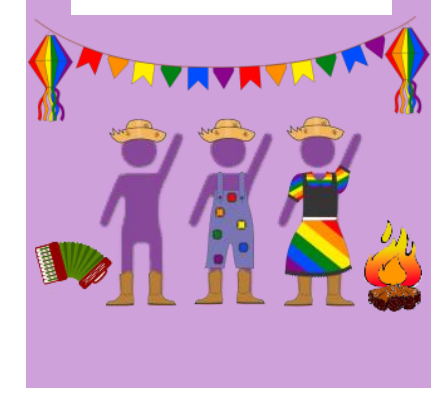

\title{
Exclusión por Estereotipos de Género en la Interacción Social Infantil
}

Jessica JAZMÍN RIVADENEIRA-PEÑAFIEL, Universidad Politécnica Salesiana del Ecuador

Daniel Llanos ERAZO, Universidad Politécnica Salesiana del Ecuador

Resumo: La exclusión es una problemática latente en la sociedad en sus distintos ámbitos y puede analizarse desde varias perspectivas, en este caso, desde una mirada decolonial que aborda el género en un entorno socioeducativo. El presente artículo tiene por objetivo analizar los procesos de exclusión por género que se producen en un grupo de estudiantes del cuarto año de Educación General Básica en un centro educativo de la ciudad de Quito, Ecuador. La metodología empleada en el estudio se enmarca en la tradición cualitativa, optando como técnicas de recogida de información, la observación participante y las entrevistas semiestructuradas. Entre los principales hallazgos, podemos afirmar que la exclusión entre pares por género persiste en las prácticas de socialización. Los elementos dinamizadores de este proceso son las narrativas y discursos que los/as docentes emplean y los preconceptos que se encuentran afincados en las prácticas lúdicas y cotidianas de los niños/as.

Palavras-Chave: Estereotipos De Género; Exclusión Social; Infancia; Interacción Social; Pensamiento Decolonial. 


\section{Introducción}

La escuela como institución social produce y reproduce características de la sociedad, algunas consideradas "positivas" como "negativas" dependiendo de los anteojos que se empleen. Entre las principales problemáticas que encontramos al interno de las escuelas es el proceso de exclusión entre pares, proceso que pulula en todos los segmentos poblacionales sin considerar el rango etario o el estrato socio económico.

En el presente artículo procuraremos analizar la influencia de la práctica docente en los procesos de inclusión/exclusión en estudiantes de cuarto año de Educación General Básica de una institución educativa particular de la ciudad de Quito, Ecuador. Cabe mencionar que los insumos y reflexiones son el resultado de un trabajo investigativo que se ejecutó entre los años 2019-2020.

Entre los principales resultados, se evidenció la existencia de procesos de exclusión por cuestiones de género entre los/as estudiantes donde se observó la presencia de estereotipos y la influencia de la práctica docente en dichos procesos debido a que la mayor parte del tiempo que los/as estudiantes están en la institución educativa, lo comparten con el/la docente. La importancia de analizar la relación que existe entre estas dos categorías (estereotipos y práctica docente) recae en que las experiencias que se adquieren en los primeros años de la vida escolar repercuten en el desarrollo y adultez de la persona.

Por esa razón, en el nivel de Educación General Básica existe una mayor responsabilidad docente en la formación del/a estudiante y por lo tanto la construcción de la ciudadanía, pues es una edad crucial en la vida de toda persona por ser los primeros años de inserción en el mundo social y comprensión de contextos que se mantienen permanentemente cambiantes.

En el presente trabajo, se presentarán algunos de los resultados obtenidos en la investigación mencionada con el objetivo de analizar los procesos de exclusión en infancias desde una mirada decolonial que aborda las diferencias de género presentes en la interacción social. Esto debido a la problemática que representa la reproducción de estereotipos de género en edades tempranas que luego se reflejarán en una sociedad que continúa con costumbres, prácticas tradicionalmente excluyentes.

Los resultados que se presentarán a continuación son producto de un estudio de caso descriptivo que utilizó una metodología cualitativa que se sirvió de preceptos provenientes del interaccionismo simbólico para 
comprender la significación de los actos humanos y la realidad del grupo social dentro de su cotidianidad, considerando todo aquello que tenga un significado simbólico en la interacción social de acuerdo al contexto en el que los sujetos se desenvuelven e interactúan.

La muestra del estudio de caso correspondió a los/as estudiantes de cuarto año de Educación General Básica que se encontraban entre los 8 y 9 años de edad. Las técnicas cualitativas que se utilizaron en la investigación fueron la observación participante y las entrevistas semiestructuradas. Sin embargo, en el presente, se analizarán únicamente los resultados obtenidos durante la observación participante por la predominancia de los procesos de exclusión por cuestiones de género evidenciados. Para complementar esta técnica, se utilizó como instrumento el diario de campo para registrar los datos relacionados al comportamiento y accionar de los/as estudiantes y docentes de la muestra.

\section{Desarrollo Interacción social}

La interacción social se entiende teóricamente como "el aspecto central de la socialización, que en síntesis puede considerarse como un proceso de relaciones a través del cual se desarrollan determinadas formas de pensar, sentir y actuar que son características de un grupo" (YUBERO, 2004, p. 799). En este proceso de relaciones es fundamental la comunicación entre las personas que están interactuando, pues es la manera en la que se conocen mutuamente para iniciar algún tipo de vínculo; ya que en ese momento "los sujetos proyectan sus subjetividades y modelos del mundo, interactúan desde sus lugares de construcción de sentido" (RIZO, 2006, p. 45).

Para que una persona se relacione al interno de un grupo social, requiere pasar por un proceso de adaptación, donde pueda identificar las pautas y códigos socioculturales del grupo, aceptarlas y seguirlas para ser parte del mismo y; además, desarrollar los cambios o adaptaciones necesarias para relacionarse de forma óptima con los demás miembros del grupo de acuerdo a las circunstancias que se presentan a la persona que ingresa al nuevo espacio de socialización (YUBERO, 2004).

Estas formas de interacción social permiten significar y a la vez re significar la realidad social de cada sujeto en un contexto determinado. En ese sentido, la interacción entre sujetos se encuentra mediada por las condicionantes socioculturales propias del contexto de origen de cada 
interactuante y a la vez, por las condiciones prevalentes en el contexto de interacción in situ.

\section{Socialización entre Pares}

La socialización de los individuos se produce en todos los contextos posibles de interacción debido a que "puede decirse que el ser humano desde que nace es un ser social, destinado a vivir en un mundo social y que, para ello, necesita la ayuda de los demás" (YUBERO, 2004, p. 796). Por tanto, cuando se habla de socialización en un ámbito educativo, ésta se presenta en todos los niveles y; en este caso, se hará énfasis en aquella que se produce en un contexto educativo entre niños/as y adultos.

A saber que "ese ser social, además, se va haciendo poco a poco a través de la interacción con los otros, en un proceso continuo de socialización" (YUBERO, 2004, p. 796), es necesario tener en cuenta la relevancia de la interacción en la formación de cada individuo como parte de una sociedad; pues, es ahí donde se descubre su "lugar" en un grupo en relación a sus experiencias con sus semejantes. Dicha interacción se produce, por lo general, con personas de características similares que pueden incluir aspectos de edad, intereses o roles en un grupo social, dándose así una socialización entre pares o también considerada como interacción social de tipo horizontal.

\section{Socialización Infantil}

Para referirnos a la socialización infantil, es necesario comprender a la infancia como aquel espacio de tránsito que provee a los sujetos de insumos sociales y culturales para una futura vida adulta. En ese sentido, la infancia como etapa importante del desarrollo se constituye en un punto de encuentros con los suyos -familia- y con los otros -paresque contribuyen, a partir de los actos socializantes, a la construcción del sujeto niño que se deviene en adulto (LLANOS e SÁNCHEZ, 2016; MIGUEL, 2014). A partir de esta etapa del ciclo vital del ser humano, emerge con fortaleza el proceso de socialización, proceso que se produce en dos espacios fundamentales, los mismos que contribuyen de forma significativa en el desarrollo de los niños/as:

El primero, es el espacio familiar, lugar en el cual el/la niño/a interactúa con sus consanguíneos y adquiere los primeros valores, normas de comportamiento y de relacionamiento social. En esa medida, la familia se constituye en el primer espacio de encuentro y de intercambios del/a 
niño/a con los “otros”. Este encuentro le permite al niño poner en práctica los primeros aprendizajes adquiridos en el seno familiar.

El segundo espacio de socialización es el educativo. Debemos comprender que el espacio escolar, como problema sociológico y como categoría analítica, constituye un hito fundamental en los estudios del vasto campo de la educación durante las primeras décadas del siglo XX. Es Emilio Durkheim, precursor de la sociología de la educación, quien definió a la educación como espacio de socialización del/a niño/a (UNDA et al., 2015).

Esta precisión durkheniana, obedeció a que durante el siglo XX la ampliación de los derechos ciudadanos permitió que millones de niños asistan al sistema educativo, deviniendo con ello, en el incremento de posibilidades socializadoras y por supuesto, la adquisición de nuevos aprendizajes.

Pero siguiendo los axiomas de Durkheim podemos afirmar que todo proceso educativo (comunitario, familiar, laboral o escolar) se encuentra atravesado por un sistema de valores, creencias y códigos de relacionamientos vivenciales, los mismos que son transmitidos e inculcados con el firme propósito de aportar en la construcción tanto del sujeto individual como del sujeto social y colectivo (sociedad).

Bajo estos presupuestos sociológicos, la idea de lo social y del sujeto social encuentra dos posibles variantes generales según tipo de sociedad: la sociedad societal y la sociedad comunal (ÁLVARO, 2010) en las que, independientemente de sus características particularidades y especificidades, operan procesos de transmisión y adquisición sociocultural y, más exactamente, educativos. En el caso que nos ocupa, abordaremos los procesos de exclusión que se producen en los espacios de interacción y socialización de niños/as en el espacio escolar.

Por todos es conocido, que la escuela como institución concentra, produce y reproduce un conjunto de problemáticas que son parte de la sociedad en su conjunto, en ese sentido, la socialización infantil pone de manifiesto los valores y creencias que aún persisten unas nuestras sociedades en pleno siglo XXI.

Si bien las políticas educativas de los Estados a nivel mundial han tenido un significativo avance en términos de inclusión social, procurando que la exclusión se elimine de forma progresiva, también es cierto que las brechas inclusivas son tareas pendientes en nuestras cotidianidades. Parecería extraño que un mundo de interacciones veloces y constantes intercambios en los cuales se supone que la pluralidad es el principio rector de toda interacción, continúen las exclusiones entre sujetos. En el 
caso de las infancias, la exclusión que prevalece es la referida al género. Este tipo de exclusión se evidencia con facilidad justamente en el proceso de socialización, es decir, los valores, las creencias y los significados que los niños tienen acerca de las niñas son expresados, de formas recurrentes, en la interacción y con mayor énfasis durante las actividades de recreación y ocio.

\section{Reproducción social}

Los procesos de interacción social tienen una influencia directa por el contexto social de origen y por la socialización propia que cada sujeto desarrolla en su entorno; en ese sentido, la reproducción social se constituye en un elemento sustantivo a la hora de explicar las visiones y perspectivas que cada sujeto posee del mundo que lo rodea, es decir, asimilando y reproduciendo lo aprendido, lo observado en los sujetos cercanos y próximos que intervinieron durante su socialización primaria y secundaria. Esto se debe a que, gracias a la socialización, una persona aprende: “a) las conductas sociales consideradas adecuadas dentro del contexto donde se encuentra el individuo en desarrollo junto con b) las normas y valores que rigen esos patrones conductuales" (YUBERO, 2004, p. 796).

La reproducción social está ligada al aspecto educativogeneracional, pues "la educación es la principal responsable de la continuidad social y sin duda, gracias a ella es posible el mantenimiento de la sociedad" (YUBERO, 2004, p. 799). A través de ella, se busca transmitir aquellos saberes y valores sociales que pasan de generación en generación gracias a los diversos procesos para que un individuo se adapte a un grupo social donde se produce además el intercambio de cultura entre pares. Por tanto, se afirma que "la socialización supone un proceso de adaptación a la sociedad que necesariamente es educativo, ya que implica el aprendizaje de una serie de contenidos que la sociedad ha preparado para tal fin" (YUBERO, 2004, p. 799).

Dentro de esta reproducción, se resalta el rol del/a docente por ser el sujeto educativo que interactúa con mayor frecuencia y constancia con los/as estudiantes. Es así, que se define a la práctica docente como "un conjunto de acciones, operaciones y mediaciones, saberes, sentires, creencias y poderes, que se desarrollan en el aula con un sentido educativo" (VERGARA, 2016, p. 77). Pero en un sentido más específico para el presente análisis, se entiende como las acciones, gestos, comportamientos e incluso discursos que el profesorado realiza en 
interacción con el estudiantado y por ello tiene intervención directa en la socialización estudiantil y los procesos de reproducción y exclusión social.

Cuando el/la docente tiene prácticas excluyentes, algunos educandos las reproducen para ser parte del grupo social cuyo pensamiento compartido es por lo general con matices de discriminación ya que "en la relación de interacción, cada interlocutor intenta adaptarse al comportamiento y expectativas del otro, con base en las reglas, normas y dinámicas compartidas" (RIZO, 2006, p. 45).

Si bien es cierto, "en el proceso de comunicación los sujetos proyectan sus subjetividades y modelos del mundo, interactúan desde sus lugares de construcción de sentido" (RIZO, 2006, p. 45); por ese motivo, un/a docente debe ser cauto y prudente en su práctica porque su accionar puede permitir o incluso promover la reproducción de ciertas ideas y percepciones que pueden contribuir a generar procesos de exclusión social y que los/as estudiantes continúen con dicha reproducción.

\section{Exclusión social}

La exclusión social se define a partir de la aproximación a la inclusión por la dualidad generada entre éstas; por tanto, se considera que "inclusión indica entrada, tránsito desde una exterioridad a una interioridad, adquirir la condición de pertenencia" (FERREIRA, 2011, p. 1). Mientras que de forma contraria, toma en cuenta los "procesos de exclusión social que provocan una situación de discriminación $\mathrm{y}$ marginación" (FERREIRA, 2011, p. 1). Cabe indicar que "las explicaciones para rechazar la exclusión a menudo son la forma de injusticia o daño psicológico al individuo excluido" (TENENBAUM et al., 2018, p. 2).

Con lo antes expuesto, el presente trabajo centra su interés en la exclusión social generada en un determinado grupo de niños/as dentro del espacio escolar. Para ello, partimos de la comprensión que todo proceso de exclusión social es un fenómeno dinámico, estructural, multicausal y multidimensional que limita la capacidad integradora que, tiempos atrás, se asentaba en los derechos de ciudadanía que propugnaban los Estados de Derecho (HERNÁNDEZ, 2008). Dicho fenómeno "deviene de causas estructurales y no meramente individuales o causales, es decir, por las transformaciones económicas y sociales que vienen caracterizando las sociedades del conocimiento" (HERNÁNDEZ, 2008, p. 39).

Debido al carácter estructural de la exclusión, ésta depende del contexto donde se produce porque, como se mencionó, depende de la forma en la cual está organizado el grupo social con sus respectivas pautas 
de funcionamiento y; por tanto, se relativiza según las características de dicho contexto social de acuerdo al tiempo y espacio situacional. Como consecuencia, se afirma que la exclusión social es producto de las interacciones matizadas por la estructura sociocultural, económica, política e incluso geográfica que determinan el contexto de los sujetos (HERNÁNDEZ, 2008).

La exclusión social puede producirse por un conjunto de causas gracias a la diversidad de cada individuo por lo que no existe un solo tipo de exclusión social que afecte a un grupo determinado; es más, puede generarse en cualquier contexto y por una infinidad de motivos de acuerdo a su estructura. En relación a ello, los procesos de exclusión no se centran solamente en las necesidades educativas, sino que abarcan varios ámbitos; a saber, que estos procesos son resultados de una estructura existente y reproductora en la sociedad, hay varias formas en las que pueden ser abordados de acuerdo al aspecto a considerar. Por ello, "la educación inclusiva también puede ser una vía esencial para superar la exclusión social que resulta de ciertas actitudes y respuestas ante las diferencias socioeconómicas, culturales, o de género, por nombrar algunas de ellas" (MINISTERIO DE EDUCACIÓN, 2011, p. 51).

Es así, que este fenómeno también está presente en un contexto educativo, en realidad, es en dicho ambiente donde se produce de manera más evidente por su carácter social. Por ello, "los agentes sociales implicados en la educación comparten una serie de hábitos [...] moldeada por las estructuras sociales" (SOLANO, 2008, p. 123); siguiendo la misma línea y tomando en consideración la reproducción social, gracias a la educación, un contexto educativo es un espacio que se convierte en el núcleo de desigualdades sociales que transmite e irradia dichas formas de desigualdad.

Un espacio de socialización, como el analizado en esta investigación, posibilita un ambiente donde "se establecen una serie de estructuras-relaciones sociales que pueden ampliar o debilitar los factores y las tendencias de exclusión (HERNÁNDEZ, 2008, p. 50). Dicha estructura depende de las características del contexto y de los sujetos que intervienen en él, "la exclusión es una construcción social, determinada por la respuesta de todos los agentes de la sociedad y vinculada con la posición particular en la estructura social” (HERNÁNDEZ, 2008, p. 39).

En la cotidianidad de la realidad educativa, los/as estudiantes e incluso docentes siguen reproduciendo esos procesos de exclusión por el contexto social y el sentido reproductor de la escuela como tal. Y, a su vez, esto se traduce en la interacción de los educandos debido a que "es en 
dichas relaciones, donde se producen comportamientos que tienen relación con las demandas, conflictos e influencias de la sociedad y la cultura" (ARIAS, 2009, p. 33).

\section{Exclusión por género}

La exclusión de género no hace referencia solamente a las mujeres pues en general "alude a un sistema de características socialmente construidas de hombres y mujeres que determinan las oportunidades y los niveles de acceso a diversos recursos y beneficios sociales" (MADERO, 2011, p. 136). Este sistema se basa en creencias aceptadas socialmente que se convirtieron en "concepciones tradicionales sobre los diferentes roles que les corresponde a hombres y mujeres en la sociedad y que influyen de manera notable en su forma de pensar y de vivir"

(GARCÍA, 2012, p. 1).

Este tipo de exclusión es una de las más frecuentes en cualquier contexto social por el arraigo de sus fundamentos ideológicos y a pesar, que se han legalizado e incluso penalizado varias formas de manifestación de la exclusión de género, aún hay situaciones constantes en las que se evidencian las posiciones y condiciones marcadas de género donde se caracteriza de forma estereotipada el comportamiento, pensamiento, personalidad, función y aptitudes que debe tener una persona de acuerdo al género (MONTECINO, 2006).

En ese sentido, el contexto relacional que se genera en una institución educativa también es espacio donde se evidencian procesos de exclusión; pues durante el trabajo de campo llevado a cabo en la investigación, se observaron varias situaciones en las que se generaron procesos de exclusión por género A continuación describimos algunos apuntes resultados del trabajo de observación:

En uno de los múltiples días en el centro educativo se observó que la docente necesitaba una silla para el aula de clase, ante este requerimiento, le solicitó a un niño que fuera a traerla, cuando lo hizo, una niña se ofreció a hacerlo, pero la educadora la ignoró y le dijo al niño: "vaya mijo, entre saludando y pida una silla, usted es fuerte así que vaya rapidito" (Profesora tutora).

La situación mencionada evidencia una percepción estereotipada de la "fortaleza" de un hombre en relación a la "debilidad" femenina que denota la decisión de la docente de ignorar a una niña que se ofreció a hacer lo solicitado y reforzar dicho estereotipo con la idea que el comentario transmite a los/as estudiantes. Esto bajo una justificación de 
"caballerosidad" puesto que, al regresar el niño con la silla, la docente le agradeció afirmando que es un "caballerito".

Como se indicó previamente, la práctica docente es un ejercicio determinante en estos procesos de exclusión social porque pueden contener acciones o comentarios que, aunque "pasen desapercibidos" por su repetición constante, naturalización, contienen rasgos estereotipados que evidencian una desventaja no solo hacia las mujeres, sino que, a nivel general, refuerzan los roles de género, los cuales, a su vez, dan paso a que puedan generarse procesos de exclusión.

Otro ejemplo registrado en los diarios de campo fue un comentario realizado por el docente, quien mencionó: "pase una niña a escribir los ejercicios en la pizarra, ellas tienen la letra más bonita" (Profesor de Educación Física). Al finalizar la clase, con la intención de llevarse un cuaderno de muestra para los otros salones, dijo en voz baja: "las niñas siempre hacen más ordenadito, pidámosle a una el cuaderno" (Profesor de Educación Física). Estos comentarios vertidos por el docente demuestran una concepción de que una mujer, en comparación a un hombre, es más "ordenada" y con actitudes más estéticas como aquellas reflejadas en la caligrafía o la presentación del cuaderno como en estos casos en particular.

Asimismo, cuando se organizaron los partidos de fútbol en la unidad educativa, el encuentro deportivo de los niños se realizó primero en la cancha grande con arcos proporcionales a su estatura, participaron los miembros de la comunidad educativa como público que presenciaba el cotejo deportivo. Mientras que, después y con escaso público, se llevó a cabo el encuentro de las niñas en la cancha pequeña con sus respectivos arcos, esta actividad se desarrolló en esta cancha a pesar de estar desocupada la cancha grande.

Detrás de estos hechos, que pueden ser anecdóticos, está la idea de que los hombres tienen más habilidades para los deportes y, en este caso particular y principal, para el fútbol por lo que se da una evidente prioridad al partido en el que intervienen los niños. Además, se consideran en desventaja las capacidades físicas de las niñas al asignarles la cancha pequeña por su capacidad de correr o de resistencia aparentemente reducida en comparación a los niños cuando según los registros en los diarios de campo, las niñas del partido demostraban más velocidad y resistencia que los niños en cuestión.

Por esa razón, desde las teorías feministas y de forma particular la pedagogía feminista busca valorizar los derechos de todas/os en los distintos aspectos de la experiencia educativa, tanto en lo extracurricular 
como en el caso evidenciado como en lo curricular. De ese modo, bajo esta mirada de la pedagogía feminista, se busca "construir currículos que reflejen, de forma equilibrada, tanto la experiencia masculina como la femenina" (DA SILVA, 1999, p. 49). Esto dado que aún prevalece la idea que las mujeres deben realizar determinadas actividades que no pueden realizar los hombres y viceversa, generando exclusión y superioridad en las relaciones de género presentes en la educación.

Esta definición de roles y funciones se encuentra delimitada de forma fáctica y tácita en el contexto ecuatoriano, manteniendo la diferenciación del papel del hombre y la mujer. Un caso ejemplificador de esto es cuando un grupo de estudiantes estaban jugando en el recreo "al papá y a la mamá”, que consiste en un juego de roles en el que los/as niños simulan que son miembros de una familia y a cada uno se le asignaba un rol específico que tienen que desempeñar en diversas situaciones familiares.

En dicho juego, un niño quiso encargarse de dirigir la actividad lúdica y designar los roles a sus compañeros/as, mismos que se definieron de la siguiente manera y en este orden: al niño que asumió el papel de padre, le solicitó ser "la cabeza" de familia y el encargado de trabajar como ingeniero para cubrir los gastos del hogar; a la niña que desempeñaba el rol de madre, le asignó la función de ama de casa o de responsable de las labores domésticas; y, a los otros dos niños, les dijo que serían los hijos de la pareja. Las acciones a realizar eran de forma espontánea, pero, a nivel general, consistía en que la mamá cuidaba a los hijos, realizaba las labores del hogar y esperaba la llegada del papá a la vivienda para proporcionarle los alimentos mientras él descansaba.

Al finalizar esa primera etapa del juego, decidieron cambiar los roles y ahora uno de los hijos decidió cambiar su papel con el del padre mientras que los/as demás lo conservaron igual. Sin embargo, la niña quería cambiar de función y sugirió que ahora ella sería quien trabaje y sea la ingeniera, pero el niño con el rol de padre se negó justificando que él no iba a ser quien realice las labores domésticas y otro niño afirmó que podrían trabajar ambos pero que no sea ingeniera sino profesora.

Ante esto, la niña afirmó que no quería ser docente sino ingeniera y el niño que asumió el rol de padre se negó diciendo que el ingeniero iba a ser él y que, si la niña no quiere ser maestra, entonces sea ama de casa. Esta perspectiva denota la idea que las labores domésticas son "propias" de las mujeres, así como algunas profesiones como la docencia, principalmente enfocada en la infancia, por un aparente "sentido materno" para tratar con niños/as. 
Mientras que los hombres ocupan el lugar de proveedor del hogar, dirigente de familia y tienen profesiones socialmente consideradas como más importantes o difíciles. Además, se resalta el hecho de que un niño fue el encargado de dirigir y asignar los roles quien, a su vez, lo hizo con un orden jerárquico liderado por el padre en la actividad lúdica. Asimismo, se destaca la poca atención otorgada a la participación y opinión de la niña durante la actividad lúdica.

Tal situación es muestra de la permanencia de los roles de género en situaciones cotidianas que también se reflejan en las elecciones profesionales $\mathrm{u}$ ocupaciones laborales. Pues, como argumenta (MONTERO, 2000), la sociedad tiene la idea errónea de que cada género posee "talentos naturales" por lo que se cree que ciertas profesiones y funciones laborales y/o domésticas son femeninas mientras que otras son masculinas.

Incluso algunos estudios han demostrado que las mujeres "se decantan por estudios relacionados con profesiones que implican la ayuda a otras personas (profesiones consideradas típicamente femeninas)" (NAVARRO e CASERO, 2012, p. 127) y; por el contrario, los hombres "son mayoría al elegir profesiones técnicas que a su vez son las más valoradas socialmente y mejor remuneradas" (NAVARRO e

CASERO, 2012, p. 127).

Así, se evidencia que estas percepciones se transmiten a través de la educación y; para esto, los principales encargados son los/as docentes por la gran cantidad de tiempo que conviven con los/as estudiantes; por ello, es imprescindible recordar que:

Las percepciones están impregnadas de prejuicios, esto es, de opiniones previas, acerca de algo que se conoce superficialmente. También cada uno se maneja con estereotipos que son imágenes mentales muy simplificadas y con pocos detalles acerca de un grupo de gente que comparte ciertas cualidades, características y habilidades. El término se usa a menudo en sentido negativo. Así todas las ideas estereotipadas sobre género, culturas, razas, clases sociales, religiones son heredadas de la cultura y aprendidas familiar y socialmente, e influyen tanto en la forma de interactuar con las personas, como en la forma en que se concibe cada uno frente a ellas (MINISTERIO DE EDUCACIÓN, 2011, p. 15).

Además, estos estereotipos también se producen porque "las percepciones y modelos mentales son el resultado de todas las experiencias que las personas han vivido u observado en los diferentes ambientes por los que pasaron desde que nacen" (MINISTERIO DE EDUCACIÓN, 2011, p. 17). 
Esto se relaciona con la reproducción social ya que los estereotipos son producto también "de lo que escuchan decir a sus padres sobre las personas y grupos, así como de lo que les llega desde los medios de comunicación" (MINISTERIO DE EDUCACIÓN, 2011, p.

17).

\section{El Género desde una Mirada Decolonial}

Con el propósito de explicar el fenómeno de la exclusión infantil, acudiremos al dispositivo teórico proveniente de la perspectiva decolonial. Entendiendo que la colonialidad presente en cada relación social de los sujetos es heredada de los procesos sociales originarios de nuestras sociedades, sociedades que fueron colonizadas no únicamente en términos políticos y geográficos (colonialidad del poder), sino y, sobre todo, por el saber. La expresión más potente de la eficacia del pensamiento científico moderno es lo que puede ser descrito literalmente como la naturalización de las relaciones sociales (LANDER, 2000). Relaciones que se encuentran normadas y establecidas por principios hegemónicos que ubican en el plano social, a unos de forma superior que otros.

Esta idea de superioridad que se encuentra instalada en la sociedad tiene su génesis en la transmisión valórica que los/as niños/as adquieren durante su proceso socializador. Ahora bien, comprender que la colonialidad del poder y del saber es la síntesis de las relaciones políticas, económicas y culturales en América Latina (QUIJANO, 2014) nos permitirá clarificar, de alguna manera, cómo los/as niños/as en su proceso de socialización continúan reproduciendo patrones culturales propios de la colonia.

Parece inaudito que en pleno siglo XXI los patrones culturales de raigambre colonial aún persisten, sin embargo, en el ejercicio investigativo pudimos identificar que en el espacio escolar las ideas de superioridad masculina continúan siendo las más habituales en los procesos socializadores tal cual se narra líneas arriba. Quizá, el trabajo educativo que se realiza en los hogares como en las escuelas no consideran la importancia de revertir las ideas sexistas y esto contribuye a la constante reproducción machista que provoca procesos de exclusión hacia las niñas como sujetos y hacia lo femenino como prácticas.

Es importante señalar que este proceso de exclusión es el resultado de epistemes y nociones que han permanecido casi inmutables a través de los siglos. En palabras de Oyèronké Oyěwùmí (2017) diríamos que la lógica cultural de las categorías sociales de occidente está basada en una ideología del determinismo biológico, determinismo que procura 
organizar las relaciones sociales y culturales a partir de las características y condiciones de la biología, dejando por fuera las prácticas sociales propias y únicas de cada sociedad. Por ello, es importante repensar desde una perspectiva crítica que el tema de género y las prácticas de exclusión por género en gran medida permanecen intactas porque en las familias como en las escuelas seguimos asumiendo los axiomas Bio-

Lógicos como las lógicas organizadoras de las relaciones.

El siglo XXI se caracteriza por ser un tiempo de reivindicaciones y triunfos en el campo de los derechos. Si bien estas victorias son el resultado de las gestas empeñadas durante las centurias que le antecedieron, también es cierto que el reconocimiento social enmarcado en la legalidad a través de políticas públicas ha sido más efectivo en estos últimos 20 años.

Los Estados Latinoamericanos en las últimas dos décadas han promovido y fortalecido los instrumentos jurídicos nacionales con el propósito de garantizar el ejercicio y cumplimiento de los derechos individuales y colectivos. En esa dirección el sistema de Naciones Unidas y diferentes organismos internacionales han jugado un importante papel en el fortalecimiento de estas acciones a nivel internacional.

En el año 2015, con la finalidad de evaluar los avances de los Estados en el campo de los derechos civiles, jurídicos, sociales, económicos y culturales de las mujeres, se decidió establecer compromisos que permitan combatir las desigualdades y todo tipo de violencias en contra de las mujeres en los países que son parte del sistema de Naciones Unidas. Empero de los compromisos acordados y de la puesta en marcha de normas jurídicas y establecimiento de nuevas políticas que aseguren los compromisos suscritos, la realidad supera las buenas intenciones y las declaraciones.

En el caso que nos ocupa abordaremos una problemática que se enmarca en el espacio escolar, lugar en el cual las desigualdades persisten y en temas de género se profundizan. La exclusión entre pares a partir de la noción de género es recurrente. El cuerpo femenino es objeto de burla y en algunas ocasiones de ludibrio por parte de los compañeros.

Pero si bien el género es un factor determinante por el cual se produce exclusión entre pares, también debemos recordar que las características asociadas al origen étnico o a las condiciones socioeconómicas son factores que producen exclusión, en ese sentido, la interseccionalidad se constituye en un elemento clave para explicar que los procesos de exclusión son el producto de la colonialidad que se instauró en el continente hace más de cinco siglos. 
La herencia colonial dejó instalada preconceptos que atraviesan las nociones de etnicidad, clase social, género y en el caso que nos ocupa etario. Si una persona es indígena o afrodescendiente, mujer, empobrecida y joven, es casi seguro que la propia sociedad se encargará de excluirle. Por ello, el tema que ponemos como punto focal de análisis permite visualizar que en un centro educativo de la ciudad de Quito estas prácticas se encuentran presentes en todos los agentes educativos (profesores/as, niñas/os y por supuesto en los padres y madres de familia).

\section{Consideraciones Finales}

La exclusión social desmantela todos los ideales de solidaridad y respeto a la diversidad pues; si se pretende igualdad de oportunidades para todos y equidad de derechos, la exclusión encuentra su categorización en la ausencia de éstas en las instituciones, en especial la escuela y la familia. Dicha ausencia a la par homogeniza a las personas y deja de lado las potencialidades individuales, es por ello que "aunque todos lo lamenten, los lazos de solidaridad que nos llevan a desear la igualdad social están, al parecer, irremediablemente debilitados" (DUBET, 2016, p. 14).

Los procesos de exclusión tienen una presencia constante en las aulas porque, por lo general, el discurso educativo que busca combatirlos no prioriza el carácter social que estos tienen y es por ello que se dificulta la aplicación de principios direccionados a la inclusión, diversidad y perspectiva decolonial que se relaciona a la exclusión generada por cuestiones de género. Esto se dificulta aún más cuando la práctica docente continúa reproduciendo estereotipos y generando procesos de exclusión constantes entre los/as estudiantes, pasando desapercibido el rol de la escuela como institución social reproductora; pues "ni la inclusión ni la igualdad de oportunidades son realidades efectivas del mundo en el que vivimos, pues vivimos en un mundo estructuralmente excluyente y desigualitario" (FERREIRA, 2011, p. 2).

Partiendo de ello, se busca encaminar la problemática hacia una mirada desde la alteridad, definida por Sousa como "el principio filosófico de alternar o cambiar la propia perspectiva por la del otro, considerando y teniendo en cuenta el punto de vista, la concepción del mundo, los intereses, la ideología del otro" (CÓRDOBA e VÉLEZ-DE LA CALLE, 2016, p. 1003). Sin olvidar que no se debe asumir o dar por sentado que la perspectiva propia es la única válida y teniendo en cuenta que "la sociedad tendrá oportunidad de disminuir la exclusión cuando practique 
una actitud de respeto hacia la identidad y accionar de los demás" (RIVADENEIRA-PEÑAFIEL, 2019, p. 42).

Como se ha mencionado a lo largo del escrito, los procesos de exclusión por cuestiones de género se encuentran presentes en la socialización cotidiana de los niños. La investigación da cuenta que esta problemática analizada a partir de la perspectiva decolonial está latente en la sociedad y se reproduce de forma constante en la interacción entre los/as estudiantes por la función socializadora y de encuentros de culturas y de condiciones infantiles diversas que se conjugan en el espacio escolar.

Dichos procesos de exclusión no solo se generan por las ideas que los/as estudiantes tienen como producto de su bagaje sociocultural por una sociedad históricamente excluyente sino también por la influencia de la práctica docente en las situaciones de socialización, influencia reflejada en narrativas y discursos cargados de preconceptos y estereotipos que naturalizan la exclusión en la cotidianidad de los/as estudiantes, quienes continúan reproduciéndola. Esta realidad se problematiza debido a la mediación docente en la interacción social infantil y la alta cantidad de tiempo que conviven con los educandos.

Por ello, los/as maestros/as deben asumir responsablemente su rol como parte de la función social propia de la escuela para combatir esta problemática no solo en el ámbito de género sino en relación a la diversidad en general por las múltiples dimensiones humanas que nos conforman como seres diferentes. Puesto que se ha evidenciado que, en la realidad educativa, los/as docentes favorecen la reproducción de estereotipos que generan a su vez procesos de exclusión y, en lugar de eso, deberían hacer uso de "las oportunidades que nos ofrece la diversidad cultural como elemento de intercambio fructífero de valores y actitudes, rompiendo prejuicios e iniciando espacios de interrelación e intercambio" (LEIVA, 2010, p. 68).

De esa manera, se busca crear "lazos e interdependencias entre los grupos culturales diversos que conviven" (LEIVA, 2010, p. 68). Esa es una forma de ir en contra de la exclusión y hay muchas maneras para promover, de acuerdo a las condiciones del momento, relaciones armónicas entre los/as estudiantes. Considerando que "aunque los procesos de exclusión se fundamenten en percepciones aceptadas socialmente, es necesario que dichas percepciones no afecten la convivencia en un ambiente diverso con seres sociales"

(RIVADENEIRA-PEÑAFIEL, 2019, p. 42). 
Es una realidad que la escuela mantiene esta desigualdad causando efectos negativos en la sociedad ya que "crecer bajo la mirada de los otros, negativa, desalentadora, indiferente [...], en fin, excluyente, no le permite a un niño o niña descubrir las propias potencialidades ni le permite experimentar su capacidad creadora" (MINISTERIO DE EDUCACIÓN, 2011, pp. 14-15). Ahí recae la necesidad de valorar la diversidad de los/as estudiantes en todos los aspectos y dimensiones humanas consolidándose en valores sociales y políticos para generar contextos educativos donde se respeten las diferencias (GALLEGO et al., 2020).

\section{Referencias}

ÁLVARO, Daniel. Los conceptos de "comunidad" y "sociedad" de Ferdinand Tönnies. Papeles del CEIC, n. 52, p. 1-24, 2010. Disponível em: $<$ https://bit.ly/3qS3Awq>.

ARIAS, Leonel. Las Interacciones Sociales que se Desarrollan en los Salones de Clase y su Relación con la Práctica Pedagógica que realiza el Docente en el Aula. Posgrado y Sociedad, v. 9, n. 2, p. 32-57, 2009. Disponível em: <https://bit.ly/3vmAoTj >.

CÓRDOBA, María e VÉLEZ-DE LA CALLE, Claudia. La alteridad desde la perspectiva de la transmodernidad de Enrique Dussel. Revista Latinoamericana de Ciencias Sociales, Niñez y Juventud, v. 14, n. 2, p. 1001-1015, 2016.

DA SILVA, Tomaz Tadeu. Documentos de Identidad. Una introducción a las teorías del currículo. Belo Horizonte: Autêntica, 1999.

DUBET, F. ¿Por qué preferimos desigualdad? Buenos Aires: Siglo XXI Editores, 2016.

FERREIRA, M. Sociedad inclusiva e igualdad de oportunidades. Ponencia presentada en Jornadas Nacionales 2011: Derecho a una vida independiente de las personas con discapacidad. Cuenca: Asociación Roosevelt. Disponível em: <https://bit.ly/2FVWIKi>. , 2011

GALLEGO, Miriam e TORRES, Wilson e RIVADENEIRA, Jessica. Acompañamiento entre pares: Una práctica de la pedagogía salesiana. Desafíos de la Educación Salesiana: Experiencias y reflexiones desde las IUS. Quito: Abya-Yala, 2020. p. 437-468. Disponível em: 
<https://bit.ly/36TWlOj>.

GARCÍA, Ramón. La educación desde la perspectiva de género. Ensayos, Revista de la Facultad de Educación de Albacete, n. 27, p. 1-18, 2012. Disponível em: <https://bit.ly/2Y8KLXr $>$.

HERNÁNDEZ, Manuel. Exclusión social y desigualdad. Murcia: Editum, 2008. Disponível em: <https://bit.ly/2Rszlfj>.

LANDER, Edgardo. La colonialidad del saber: eurocentrismo y ciencias sociales. Perspectivas latinoamericanas. Buenos Aires: CLACSO, 2000. Disponível em: <https://bit.ly/3toDtPz $>$.

LEIVA, Juan. Práctica de la interculturalidad desde la perspectiva docente: Análisis y propuestas pedagógicas. Cultura y Educación, v. 22, n. 1, p. 67-84, 2010.

MADERO, Ignacio. Inclusión y exclusión de género y clase al interior de la escuela chilena en 4 comunas del sur de Chile. Estudios Pedagógicos XXXVII, n. 2, p. 135-145, 2011. Disponível em: <https://bit.ly/32BS7qJ>.

MINISTERIO DE EDUCACIÓN. Curso de Inclusión Educativa. Programa de Formación Continua del Magisterio Fiscal. Quito: Ministerio de Educación de Ecuador, 2011. Disponível em: $<$ https://bit.ly/2wKlTKy>.

MONTECINO, Sonia. Signos de la exclusión: Las relaciones de género y el juego de loinvisible/visible. Revista Mad. Revista del Magíster en Análisis Sistémico Aplicado a la Sociedad, n. 1, p. 139-148, 2006. Disponível em: <https://bit.ly/2OhJWvM>.

MONTERO, María Teresa. Elección de carrera profesional: Visiones, promesas y desafíos. Juárez: Universidad Autónoma de Ciudad Juárez, 2000. Disponível em: <https://bit.ly/2cdO2BU>.

NAVARRO, Capilla e CASERO, Antonio. Análisis de las diferencias de género en la elección de estudios universitarios. Estudios sobre educación, v. 22, p. 115-132, 2012. Disponível em: $<$ https://bit.ly/32z54AS $>$.

OYĚWÙMÍ, Oyèronké. La invención de las mujeres. Una perspectiva africana sobre los discursos occidentales del género. Bogotá: Editorial en 
la frontera, 2017. Disponível em: <https://bit.ly/3liLiUd $>$.

QUIJANO, Aníbal. Colonialidad del poder, eurocentrismo y América Latina. Cuestiones y horizontes: de la dependencia histórico-estructural a la colonialidad/descolonialidad del poder. Buenos Aires: CLACSO, 2014. . Disponível em: <https://bit.ly/3s42Vd3>.

RIVADENEIRA-PEÑAFIEL, Jessica. La exclusión en el contexto universitario. 2019, Americana, São Paulo: UNISAL, 2019. p. 40-42. Disponível em: <https://bit.ly/2wFiQH8>.

RIZO, Marta. George Simmel, Sociabilidad e Interacción. Aportes a la ciencia de la comunicación. Cinta de Moebio, n. 27, p. 43-60, 2006. Disponível em: <https://bit.ly/2KTPPMH >.

SOLANO, Juan Carlos. La exclusión social a través de la desigualdad de oportunidades educativas. Exclusión social y desigualdad. Murcia: Editum, 2008. p. 105-130. Disponível em: <https://bit.ly/2Rszlfj >

TENENBAUM, Harriet R et al. Young people's reasoning about exclusion in novel groups. Journal of Experimental Child Psychology, v. 175, p. 116, 2018. Disponível em: <https://bit.ly/2yrohWP>.

UNDA, René e MAYER, Liliana e LLANOS, Daniel. Socialización escolar: procesos, experiencias y trayectos. Quito: Abya-Yala, 2015. Disponível em: <https://bit.ly/38LKWQV >.

VERGARA, Martha. La práctica docente. Un estudio desde los significados. Revista Cumbres, v. 2, n. 1, p. 73-99, 2016. Disponível em: $<$ https://bit.ly/34GYyM3>.

YUBERO, Santiago. Socialización y aprendizaje social. Psicología social, cultura y educación. España: Pearson, 2004. p. 796-820. Disponível em: $<$ https://bit.ly/2rrbAZb>. 


\section{Exclusão devido a estereótipos de gênero na interação social infantil}

RESUMEN/ABSTRACT: A exclusão é um problema latente na sociedade em suas diferentes áreas e pode ser analisado de várias perspectivas, neste caso, a partir de uma aparência decolonial que aborda o gênero em um ambiente socioeducativo. O objetivo deste artigo é analisar os processos de exclusão de gênero que ocorrem em um grupo de alunos do quarto ano de educação geral básica em um centro educacional da cidade de Quito, Ecuador. A metodologia utilizada no estudo faz parte da tradição qualitativa, optando como técnicas de coleta de informações, observação participante e entrevistas semiestruturadas. Entre as principais descobertas, podemos afirmar que a exclusão entre os pares por gênero persiste em práticas de socialização. Os elementos dinâmicos desse processo são as narrativas e os discursos que os professores empregam e os preconceitos enraizados nas práticas brincalhonas e cotidianas das crianças. PALABRAS ClaVE/KeYWORDS: Estereótipos De Gênero; Exclusão Social; Infância; Interação Social; Pensamento Decolonial.

Jessica JAZMÍN RIVADENEIRA-PEÑAFIEL

Universidad Politécnica Salesiana del Ecuador Licenciada en Ciencias de la Educación Básica. Universidad Politécnica Salesiana del Ecuador e-mail: jessjaz98@hotmail.es

Daniel Llanos ERAZO

Universidad Politécnica Salesiana del Ecuador Doctor en Ciencias Sociales, Profesor Investigador de la Universidad Politécnica Salesiana del Ecuador e-mail: danielgllanose@gmail.com

Recebido em: 16/o3/2021

Aprovado em: 01/o6/2021 